\title{
Matched-pair comparison of ${ }^{18} \mathrm{~F}$-DCFPyL PET/CT and ${ }^{18} \mathrm{~F}-\mathrm{PSMA}-1007$ PET/CT in 240 prostate cancer patients; inter-reader agreement and lesion detection rate of suspected lesions. \\ Maurits Wondergem ${ }^{1}$, MD, PhD, Friso M. van der Zant ${ }^{1}, \mathrm{MD}$, PhD, Wouter A.M. Broos ${ }^{1}$, MD, Remco J.J. $\mathrm{Knol}^{1}, \mathrm{MD}, \mathrm{PhD}$ \\ 1.Department of Nuclear Medicine, Noordwest Ziekenhuisgroep, Alkmaar, The Netherlands
}

Running title: DCFPyL versus PSMA-1007

Keywords:

Prostate cancer, PSMA, $\left[{ }^{18} \mathrm{~F}\right]-\mathrm{DCFPyL},\left[{ }^{18} \mathrm{~F}\right]-\mathrm{PSMA}-1007, \mathrm{PET} / \mathrm{CT}$

Corresponding author/ first author

Maurits Wondergem, MD, Nuclear Medicine Physician

Address: Noordwest Ziekenhuisgroep, Wilhelminalaan 12, 1815 JD, 0030, The Netherlands

M.Wondergem@nwz.nl

Phone: +31725483480

Fax: $\quad+31725483484$

ORCID: M. Wondergem 0000-0001-8227-9145 


\section{ABSTRACT}

Introduction

Tens of different Prostate Specific Membrane Antigen (PSMA) targeting radiopharmaceuticals for both imaging and therapy have been synthesized. Although variability in biodistribution and affinity for binding to the PSMA receptor between different PSMA targeting radiopharmaceuticals are known, little is known about the clinical implications of those variabilities. Therefore, in this study differences in interreader agreement and detection rate between two regularly used ${ }^{18}$ F-labeled PSMA-receptor targeting radiopharmaceuticals $\left[{ }^{18} \mathrm{~F}\right]-$ DCFPyL and $\left[{ }^{18} \mathrm{~F}\right]-\mathrm{PSMA}-1007$ were analyzed.

\section{Material and methods}

One hundred and twenty consecutive patients scanned with [ $\left.{ }^{18} \mathrm{~F}\right]-\mathrm{PSMA}-1007$ were match-paired with 120 patients scanned with $\left[{ }^{18} \mathrm{~F}\right]$-DCFPyL. All 240 PET/CTs were reviewed by two readers and scored according to PSMA-RADS reading criteria for PSMA PET/CT. Inter-reader agreement and detection rate of suspected lesions were scored for different anatomical locations including prostate/prostatic fossa, lymph nodes, bone, and other locations.

\section{Results}

Large equality between $\left[{ }^{18} \mathrm{~F}\right]-\mathrm{DCFPyL}$ and $\left[{ }^{18} \mathrm{~F}\right]-\mathrm{PSMA}-1007$ was found; however, some clinically relevant and statistically significant differences were observed. $\left[{ }^{18} \mathrm{~F}\right]-\mathrm{PSMA}-1007$ detected suspected prostatic/prostatic fossa lesions in a higher proportion of patients and especially in the subcohort of patients scanned for biochemical recurrence. $\left[{ }^{18} \mathrm{~F}\right]-$ DCFPyL and $\left[{ }^{18} \mathrm{~F}\right]-$ PSMA-1007 showed equal ability for detection of suspected lymph nodes, although inter-reader agreement for $\left[{ }^{18} \mathrm{~F}\right]$-DCFPyL was higher. $\left[{ }^{18} \mathrm{~F}\right]$ DCFPyL showed less equivocal skeletal lesions and higher inter-reader agreement for skeletal lesions. The majority of equivocal lesions found with PSMA-1007 (at least were determined to be aof nonmetastatic origin.

\section{Conclusion}

Clinically relevant differences, which may account for diagnostic dilemmas, were observed between of $\left[{ }^{18} \mathrm{~F}\right]-\mathrm{DCFPyL}$ and $\left[{ }^{18} \mathrm{~F}\right]-\mathrm{PSMA}-1007$. Those findings encourage further studies, as they may have consequences for selection of the proper PSMA targeting radiopharmaceutical. 


\section{INTRODUCTION}

In recent years Prostate Specific Membrane Antigen receptor (PSMA) Positron Emission Tomography/Computed Tomography (PET/CT) has rapidly evolved as a corner stone in prostate cancer imaging. PSMA PET/CT outperforms other imaging modalities due to its superior sensitivity and specificity, although sensitivity for lymph node metastases has been found to be moderate in prospective trials $(1,2,3,4,5)$. The better diagnostic characteristics account for better treatment selection in both primary staging as well as biochemical recurrence. The ability to detect small metastases for example, may be beneficial for patients with oligometastatic disease offering them treatment options with chance of curation or survival benefits(6, 7), although scientific underpinning of the latter is needed(8). The additional value of PSMA PET/CT during follow-up of systemic treatment, including androgen deprivation therapy and chemotherapy, in the palliative phase of the disease also needs to be clarified.

Due to the success of PSMA PET/CT, the number of different radiopharmaceuticals targeting the PSMA-receptor has increased significantly(9). While most early publications on clinical use of PSMA $\mathrm{PET} / \mathrm{CT}$ reported on findings with ${ }^{68} \mathrm{Ga}$-labeled radiopharmaceuticals, later on ${ }^{18} \mathrm{~F}$-labeled radiopharmaceuticals also received attention. Positrons emitted by ${ }^{18} \mathrm{~F}$ decay have lower kinetic energies as compared to those emitted by ${ }^{68} \mathrm{Ga}$, which results in a higher resolution of PET images acquired using

${ }^{18} \mathrm{~F}$-tracers. Furthermore, the 110 -minute half-life of ${ }^{18} \mathrm{~F}$ compared to 68 minutes for ${ }^{68} \mathrm{Ga}$ enables imaging at later time points without significant deterioration of image quality or the need for administration of higher dosages. This is of clinical importance since PSMA-tracer kinetics show that the tracer accumulates in prostate cancer cells over time while background activity decreases $(10,11,12,13$, 14). While tens of different PSMA targeting radiopharmaceuticals for both imaging and therapy have been synthesized, only a limited number are used in common clinical practice.

Although variability in biodistribution and affinity for binding to the PSMA receptor between the different PSMA targeting radiopharmaceuticals are known, little is known about the clinical implications of those variabilities $(15,16)$. This is reflected in recent guidelines which do not discriminate between different PSMA-targeted radiopharmaceuticals for imaging of prostate cancer (https://uroweb.org/guideline/prostate-cancer/). To investigate whether abovementioned differences are of clinical importance and interfere with reproducibility of scan outcomes and lesion detection, in this study inter-reader agreement and detection of suspected lesions of two regularly used ${ }^{18} \mathrm{~F}$-labeled PSMA-receptor targeting radiopharmaceuticals, 2-(3-(1-carboxy-5-[(6-[ $\left.{ }^{18} \mathrm{~F}\right]$ fluoro-pyridine-3-carbonyl)- 
amino]-pentyl)-ureido)-pentanedioic acid (DCFPyL) and ((3S,10S,14S)-1-(4-(((S)-4-carboxy-2-((S)-4carboxy-2-(6- ${ }^{18}$ F-fluoronicotinamido)butanamido)butanamido)methyl)phenyl)-3-(naphthalen-2ylmethyl)-1,4,12-trioxo-2,5,11,13-tetraazahexadecane-10,14,16-tricarboxylic acid) (PSMA-1007), were analyzed in a large matched paired cohort of 240 patients. Known differences between those two specific radiopharmaceuticals, that may interfere with scan readability, include differences in excretion pathways and bone marrow uptake $(15,16)$. Renal excretion of DCFPyL results in high activity in the urinary tract, which may interfere with detection of lesions in the proximity of ureters and urinary bladder, while activity in the urinary tract is usually less for PSMA-1007; due to biliary excretion as the most important excretion pathway. Physiological bone marrow uptake of PSMA-1007 is commonly higher, which may interfere with detection of bone metastases.

\section{Material and methods}

Patient population

One hundred and twenty consecutive patients imaged between $2^{\text {nd }}$ April 2019 and $20^{\text {th }}$ June 2019 with PSMA-1007 PET/CT for primary staging, biochemical recurrence, or follow-up of systemic treatment of prostate cancer were retrospectively included in the study. In order to create match pairs 120 matched patients scanned with DCFPyL were extracted from a prospectively maintained database consisting of 813 patients scanned with DCFPyL between $3^{\text {th }}$ November 2016 and $21^{\text {st }}$ March 2019. Matched pairs were created to allow for a fair comparison of the cohorts. Pairs were matched on basis of disease stage (primary staging, biochemical recurrence or treatment follow-up of castrate resistant prostate cancer), Prostate-specific Antigen (PSA) at time of PET/CT (PSA difference between two patients $<10 \%$ ) and, in case of biochemical recurrence, previous treatment (prostatectomy, lymph node dissection, external radiation therapy or brachytherapy).

Besides the standard PSMA PET/CT, acquired on clinical indication, no additional measurements or actions affecting the patient were performed. The study passed the local scientific board and approval of the local ethical committee for the present study was waived since the study does not fall within the scope of the Dutch Medical Research Involving Human Subjects Act (section 1.b WMO, 26 ${ }^{\text {th }}$ February 1998). Additionally, as a standard procedure on our department, all included patients gave written consent for usage of their anonymized data for scientific purposes. 
Image acquisition

DCFPyL and PSMA-1007 were synthesized by an on-site cyclotron facility. One hundred and twenty minutes post injection of ${ }^{18} \mathrm{~F}$-DCFPyL (mean $319 \mathrm{MBq}, 231-367$ range; depending on body mass) and 90 minutes post injection of PSMA-1007 (mean 324 MBq, 239-363 range; depending on body mass), PET images were acquired on a Siemens Biograph-16 TruePoint PET/CT (Siemens Healthcare, Knoxville, U.S.). Images were acquired from the inguinal region to the base of the skull (5 minutes per bed position). Data was reconstructed using an iterative ordered-subset expectation maximisation-3D algorithm; 4 iterations, 16 subsets and $5 \mathrm{~mm}$ Gaussian filter. Image matrix size was $256 \times 256$, pixel spacing $2.67 \times 2.67 \mathrm{~mm}$ and slice thickness $4 \mathrm{~mm}$. For attenuation correction a radiocontrast enhanced CT (110 mAs at 110-130 kV) was typically acquired. Collimation was $16 \times 1.2 \mathrm{~mm}$, pitch 0.95 , slice thickness $4 \mathrm{~mm}$ and matrix size $512 \times 512$. This resulted in voxel sizes of $1.37 \times 1.37 \mathrm{~mm}$ for CT images for attenuation correction and $0.98 \times 0.98 \mathrm{~mm}$ for diagnostic CT images.

Data acquisition

For this study all 240 included PET/CTs were re-evaluated by two Nuclear Medicine physicians (FZ and MW) with ample experience in reading of both DCFPyL and PSMA-1007 PET/CT (each reader >300 readings of both DCFPyL and PSMA-1007). Readers had access to limited patient data including: clinical indication for PSMA PET/CT, PSA at time of PSMA PET/CT, Gleason score, TNM-stage as far as known before PSMA PET/CT and previous treatments received by the patient. Readers were blinded for all other data, including the used radiopharmaceutical.

Scan outcome was scored for different anatomical localizations including: prostate/prostatic fossa, inguinal lymph nodes, pelvic lymph nodes (N1-nodes, according to TNM $8^{\text {th }}$ edition of the American Joint Committee on Cancer), abdominal lymph nodes, thoracic lymph nodes, axillary lymph nodes, cervical lymph nodes, pelvic bones, vertebral bones, thoracic bones (costae, sternum, claviculae and scapulae), bones of the extremities and other suspected lesions. PSMA PET/CTs were read using the PSMA-RADS criteria, which are specifically determined for reading of PSMA PET/CTs(17). For each reading system and each anatomical localization scan outcomes were entered in a database. Thereby only the lesions with the highest score/highest likelihood of malignancy according to the reading system were recorded. For patients with only equivocal lesions outside the prostate / prostatic bed by one or both readers, the true nature of the equivocal finding was retrospectively determined using data from: histopathological biopsies, imaging at follow-up or PSA response to therapy. Definitive proof of nonmetastasized disease included: histopathological findings excluding metastasized disease, complete biochemical response after local therapies including prostatectomy, lymphadenectomy, radiation 
therapy without or after discontinuation of androgen deprivation therapy without local therapy of the equivocal findings. Definitive proof of metastasized disease included: concordant findings at MRI, progression of lesions on follow-up PSMA PET/CT or PSA response to targeted therapy on equivocal findings.

Statistical analysis

The Mann-Whitney $U$ test was used to test for differences between DCFPyL and PSMA-1007 outcomes. Inter-reader agreement was measured by calculating the weighted Cohen's Kappa (weighted к) and the percentage of agreement. As conventionally done, agreement was divided into the categories: poor, fair, moderate, good, and very good, reflecting agreement of 0-19, 20-39, 40-59, 60-79 and $80-100 \%$, respectively. For weighted $\mathrm{k}$ it must be noted that weighted $\mathrm{k}$ is highly dependent on the proportion of positive scan results, thereby a low weighted $\mathrm{k}$ may reflect good or even very good agreement when proportions of positivity are relatively low (app. <0.2) or high (app. >0.8)(18). For calculation of differences between DCFPyL and PSMA-1007 and agreement between readers, some subcategories of the PSMA-RADS reading system were merged since some subdivisions barely have clinical impact; $1 a, 1 b$ and 2 (benign and likely benign), 3a, 3b, 3c, and 3d (equivocal) and 4 and 5 (likely malignant and malignant). Pie plots were constructed for outcomes that showed relevant differences. All analyses were performed using a Statistical Package for Social Sciences (IBM SPSS Statistics 25.0, Chicago, IL, USA).

\section{RESULTS}

A total of $240{ }^{18} \mathrm{~F}$-PSMA PET/CT scans, 120 DCFPyL and 120 PSMA-1007, were included in the study (Table 1). Commonly found differences between DCFPyL and PSMA-1007 uptake are shown in Figure 1.

Prostate / Prostatic fossa

For the prostate/ prostatic fossa, inter-reader agreement was very good for DCFPyL and good for PSMA-1007 (Figure 2). Both readers found significantly more suspected prostate lesions with PSMA1007 using the PSMA-RADS criteria (Table 2, Supplement 1). Categorization of the cohort according to disease stage shows statistical differences for detection of suspected prostate lesions by both readers for patients with biochemical recurrence only (Table 3). 
Lymph nodes

Inter-reader agreement for lymph nodes was in general good to very good for both DCFPyL and PSMA-1007. Although a consistently lower agreement, for both weighted $\mathrm{k}$ and percentage of agreement, was found for detection of suspected pelvic lymph nodes and lymph nodes at any localization with PSMA-1007. No statistically significant differences were found between DCFPyL and PSMA-1007 for detection of suspected lymph nodes (Table 2). However, with PSMA-1007 more lymph nodes showed low level uptake comparable to blood pool activity, matching score $1 \mathrm{~b}$ or 2 (benign or likely benign) (Supplement 2). For most anatomical regions significantly more lymph nodes with low PSMA-1007 uptake were found compared to DCFPyL $(p<0.0005-0.037)$.

Bone lesions

Regarding suspected bone lesions almost perfect inter-reader agreement was found at all localizations for DCFPyL. For PSMA-1007, however, consistently lower agreement (both weighted $\mathrm{k}$ and percentage of agreement) was found for lesions in the thoracic region and whole skeleton and to a lesser extent for suspected bone lesions in the pelvis (Figure 2). Both readers scored significantly more equivocal bone lesions in the thoracic region with PSMA-1007 (14-33\% and 3-4\% for PSMA-1007 and DCFPyL, respectively) at the expense of the number of scans without bone lesions (44-62\% for PSMA1007 and 76-78\% for DCFPyL). The same is found for bone lesions at any localization (equivocal: 14-33\% PSMA-1007 and 3-4\% DCFPyL; no bone lesions 30-44\% PSMA 1007 and 64-66\% DCFPyL) (Table 2, Figure 3). Reader 2 also scored significantly more equivocal pelvic lesions ( $p=0.027)$, while for reader 1 no statistically significant difference was found $(p=0.053)$. Categorization according to disease stage shows that significantly more equivocal lesions are found with PSMA-1007 at primary staging and biochemical recurrence $(P=0.001$ and 0.003$)$ also at the expense of the number of patients without bone lesions (Table 3).

Visceral lesions

Visceral lesions were found in 3\% of patients by both readers for both PSMA-1007 and DCFPyL, while equivocal lesions were found in 5-6\% of patients with DCFPyL and $8 \%$ of patients with PSMA-1007 (Table 2). No statistically differences were found between PSMA-1007 and DCFPyL for detection of other lesions. All patients that showed suspected lesions at other locations had extensive metastasized disease in lymph nodes and skeleton. 
Equivocal bone lesions

Three patients scanned with DCFPyL (1 primary staging, 1 BCR, and 1 therapy follow-up) and 26 scanned with PSMA-1007 (18 primary staging, 6 BCR, and 2 therapy follow-up) had equivocal bone lesions as only indication of possible metastasized disease. Clinical follow-up of those patients showed definite proof of non-metastasized disease in 0 and 16 (62\%) of patients scanned with DCFPyL and PSMA-1007, respectively, while $1 \%(33 \%)$ and $2(8 \%)$ showed proof of metastasized and no conclusive follow-up was present in 2 (67\%) and 8 (31\%). From 3 patients histopathological verification of equivocal bone lesions on PSMA-1007 was present; of those lesions none have been proven to be malignant (Figure 4). Histopathology showed benign etiologies including aspecific necrosis, aspecific lytic bone lesion with reactive changes and normal bone tissue in one case.

\section{DISCUSSION}

Although a large equality between DCFPyL and PSMA-1007 for inter-reader agreement and for detection of suspected prostate cancer lesions is found, there are two differences that may be of clinical relevance.

First the prostatic region; identification of those lesions in this region is especially of clinical importance for patients with biochemical recurrence. The sub analysis according to clinical stage showed that especially in this category of patients PSMA-1007 detects significantly more suspected lesions than DCFPyL, although the number of patients in this category is limited $(n=21)$. That finding could be the result of the lower urinary secretion of this radiopharmaceutical, allowing for better characterization of intraprostatic lesions or lesions in the prostatic fossa after prostatectomy.

Second, equivocal bone lesions were more frequently found with PSMA-1007 and resulted in lower inter-reader agreement. The greatest difference between both readers was found in the classification of rib uptake. Both readers acknowledged high uptake in ribs in a larger number of patients scanned with PSMA-1007. Reader 1 interpreted those as probably benign (PSMA-RADS 2) in a larger proportion than Reader 2, who scored those as equivocal (PSMA-RADS3a-d). Reader 1 argued that the absence of skeletal lesions at other sites in the skeleton made bone metastases in the ribs unlikely. While reader 2 scored those rib lesions as equivocal purely based on the increased activity in the ribs. Reader 2 argued that solitary bone metastases in ribs are encountered in prostate cancer patients, although, as far as known today, normally not as frequently as observed with PSMA-1007. The PSMARADS reading criteria allow for interpretation differences, since no hard cut-off values to classify tracer uptake are given. Although allowance of differences in interpretation is scientifically less desirable, they 
are continuously present in clinical practice and therefore these data reflect the presence of discussion around this topic in present daily practice. Findings of equivocal PSMA avid bone lesions may be clinically relevant and account for diagnostic dilemmas, for example in absence of other metastatic lesions or when it distinguishes between oligo- and polymetastatic disease. In these cases, the true nature of those equivocal findings may alter patients' management and further diagnostic procedures may be needed.

Given the retrospective nature of the study for almost all lesions histopathological confirmation is lacking, which is a drawback of this study. However clinical follow-up of patients with equivocal bone lesions as only indication of possible metastasized disease showed that in the majority (at least 16/24) of patients with those abnormalities on PSMA-1007, metastasized disease could be excluded. While metastasized disease was proven in only $2 / 26$ patients. Those findings provide indications that equivocal bone findings with PSMA-1007 PET/CT are of little clinical value and should not result in withholding patients from treatment options with curative intention.

As another drawback of the study DCFPyL and PSMA-1007 were compared by means of an interpatient comparison, which may result in findings that are attributable to differences between the cohorts rather than differences between both radiopharmaceuticals. However, in order the diminish these influences two large cohorts were used which comprised match-paired patients. Unfortunately, the databases were not large enough to enable pairing by all potentially relevant clinical characteristics that may interfere with the pretest likelihood to detect suspected lesions. Matching with PSA was done instead of matching regarding to Gleason score or T-stage since there are indications that ${ }^{18} \mathrm{~F}$-PSMAPET/CT positivity for metastases is best predicted by the serum PSA-value (19).

The findings in this study are in line with findings by Dietlein et al. in an intrapatient comparison of PSMA-1007 with either DCFPyL, ${ }^{68}$ Ga-PSMA-11 or ${ }^{18}$ F-JK-PSMA-7(20). In a cohort of 27 patients a better characterization of prostate lesions with PSMA-1007 was shown $(p=0.024)$ at the expense of the interpretability of skeletal lesions. PSMA-1007 showed significantly ( $p=0.0006)$ more aspecific medullary focal uptake. However, in another study that included 12 patients scanned with both DCFPyL and PSMA1007 , both radiopharmaceuticals identified exactly the same lesions and the appearance of equivocal skeletal uptake was not mentioned(15). Recently Rauscher et al. performed a matched-pair analysis of ${ }^{68}$ Ga-PSMA-11 and PSMA-1007.

They found an almost 5 times higher prevalence of PSMA-ligand-positive findings attributed to a benign origin including amongst other bone lesions and lymph nodes(21). For this study, as is the case for the present study, the lack of histopatholigical confirmation represent a major limitation. To our 
knowledge this is the first study comprising a large cohort of patients assessing the differences between two widely used ${ }^{18}$ F-PSMA radiopharmaceuticals.

The mechanism of nonspecific skeletal PSMA-1007 uptake is unknown and the appearance shows large heterogeneity between patients (Figure 1). It could be hypothesized that radiolysis may result in free ${ }^{18} \mathrm{~F}$-fluorine, however quality control reports of our cyclotron facility mentioned concentrations of free ${ }^{18} \mathrm{~F}$-fluorine below $2 \%$ at 8 hours post synthesis of PSMA-1007. Furthermore, the observed aspecific skeletal uptake is not recognized in patients with prostate cancer scanned with ${ }^{18} \mathrm{~F}$ Sodiumfluoride(22). Therefore, it is unlikely that the uptake is explained by presence of free ${ }^{18} \mathrm{~F}$-fluorine due to radiolysis. In literature, comparing PSMA-tracers with ${ }^{68} \mathrm{Ga}$ and ${ }^{18} \mathrm{~F}$, it has been suggested that the higher number of equivocal lesions found with PSMA-1007 might be explained by the lower positron energy of ${ }^{18} \mathrm{~F}$ compared to ${ }^{68} \mathrm{Ga}$, which improve spatial resolution, and the longer half-life and generally higher injected activities(21). However, since this study compared two different ${ }^{18} \mathrm{~F}$-labbeld PSMA tracers, it can be concluded that the differences in physical properties of ${ }^{68} \mathrm{Ga}$ and ${ }^{18} \mathrm{~F}$ do not present a good explanation for the found number of equivocal lesions with PSMA-1007. It is known that PSMAuptake may appear in several benign (bone) lesions(23), therefore it may be hypothesized that higher affinity of PSMA-1007 for the PSMA receptor, which is shown in preclinical studies, may result in a higher signal from those benign lesions(24). It could also be suggested that PSMA-1007 is metabolized, for example in the liver before excretion in the bile, resulting in radiolabeled-substances that accumulate at specific sites in the skeleton, although there is no scientific underpinning for this hypothesis.

Given the findings in the present study and available literature it can be concluded that there are differences between DCFPyL and PSMA-1007 that may have clinical consequences. Although there are clues that DCFPyL may be more appropriate when information about uptake in the prostate is of no clinical value, for example in primary staging of already histopathological proven prostate cancer and PSMA-1007 may be more appropriate in cases where detection of abnormalities in the prostate/prostatic fossa are of most clinical relevance, there is too little evidence to support recommendations on which tracer should be used. Further studies are needed to verify the clues found in this study and available literature; especially studies designed for analysis of the true nature of the equivocal bone lesions frequently found with PSMA-1007 and analysis of the from this study apparent better ability of PSMA-1007 to detect lesions in the prostate/prostatic region. 


\section{CONCLUSION}

Large equality between DCFPyL and PSMA-1007 was found; however, some differences were observed that may be of clinical relevance. PSMA-1007 detected prostatic/prostatic fossa lesions in a higher proportion of patients, while DCFPyL showed less equivocal skeletal lesions and higher interreader agreement for skeletal lesions. Those findings encourage further studies to evaluate the true clinical impact of the observed differences, as they may have consequences for selection of the proper PSMA targeting radiopharmaceutical.

\section{Compliance with Ethical Standards}

Funding: No funding was received for this study.

Conflict of Interest: None of the authors have conflicts of interest to declare.

Ethical approval: All procedures performed in studies involving human participants were in accordance with the ethical standards of the institutional and/or national research committee and with the 1964 Helsinki declaration and its later amendments or comparable ethical standards. The study passed the local scientific board and approval of the local ethical committee for the present study was waived since the study does not fall within the scope of the Dutch Medical Research Involving Human Subjects Act (section 1.b WMO, 26 ${ }^{\text {th }}$ February 1998). Furthermore, as a standard procedure on our department, all included patients gave written consent for usage of their anonymous data for scientific purposes. Informed consent: Need for written

QUESTION: Do differences in biological behavior of different PSMA tracers have clinical relevance according to inter-reader agreement and detection rate of for malignancy suspected lesions? PERTINENT FINDINGS: Inter-reader agreement of DCFPyL is generally better than for PSMA-1007, most obvious regarding bone lesions. Statistically more suspected lesions in the prostate/prostatic fossa are found with PSMA-1007 especially in the subcohort of biochemical recurrence $(p=0.007-0.029)$, while DCFPyL resulted in statistically less equivocal bone lesions most relevant in the subcohort of biochemical recurrence $(p=0.003-0.013)$ and primary staging $(p=0.001-0.065)$

IMPLICATIONS FOR PATIENT CARE: Although more studies are needed to further explore the exact clinical implications of present findings, those findings may result in a more proper selection of PSMAtargeted radiopharmaceuticals and therefore increase the diagnostic power of PSMA-PET/CT. 


\section{REFERENCES}

1. Afshar-Oromieh A, Zechmann CM, Malcher A, et al. Comparison of PET imaging with a (68)ga-labelled PSMA ligand and (18)F-choline-based PET/CT for the diagnosis of recurrent prostate cancer. Eur J Nucl Med Mol Imaging. 2014;41:11-20.

2. Bluemel C, Krebs M, Polat B, et al. 68Ga-PSMA-PET/CT in patients with biochemical prostate cancer recurrence and negative 18F-choline-PET/CT. Clin Nucl Med. 2016;41:515-521.

3. Corfield J, Perera M, Bolton D, Lawrentschuk N. (68)ga-prostate specific membrane antigen (PSMA) positron emission tomography (PET) for primary staging of high-risk prostate cancer: A systematic review. World J Urol. 2018;36:519-527.

4. van Kalmthout LWM, van Melick HHE, Lavalaye J, et al. Prospective validation of gallium-68 PSMAPET/CT in primary staging of prostate cancer patients. J Urol. 2019;203:537-545.

5. Gorin MA, Rowe SP, Patel HD, et al. Prostate specific membrane antigen targeted (18)F-DCFPyL positron emission tomography/computerized tomography for the preoperative staging of high risk prostate cancer: Results of a prospective, phase II, single center study. J Urol. 2018;199:126-132.

6. Roach PJ, Francis R, Emmett L, et al. The impact of (68)ga-PSMA PET/CT on management intent in prostate cancer: Results of an australian prospective multicenter study. J Nucl Med. 2018;59:82-88.

7. Calais J, Czernin J, Cao M, et al. (68)ga-PSMA-11 PET/CT mapping of prostate cancer biochemical recurrence after radical prostatectomy in 270 patients with a PSA level of less than $1.0 \mathrm{ng} / \mathrm{mL}$ : Impact on salvage radiotherapy planning. J Nucl Med. 2018;59:230-237. 
8. Ost P, Reynders D, Decaestecker K, et al. Surveillance or metastasis-directed therapy for oligometastatic prostate cancer recurrence: A prospective, randomized, multicenter phase II trial. J Clin Oncol. 2018;36:446-453.

9. Eiber M, Fendler WP, Rowe SP, et al. Prostate-specific membrane antigen ligands for imaging and therapy. J Nucl Med. 2017;58:67S-76S.

10. Szabo Z, Mena E, Rowe SP, et al. Initial evaluation of [(18)F]DCFPyL for prostate-specific membrane antigen (PSMA)-targeted PET imaging of prostate cancer. Mol Imaging Biol. 2015;17:565-574.

11. Afshar-Oromieh A, Malcher A, Eder M, et al. PET imaging with a [68Ga]gallium-labelled PSMA ligand for the diagnosis of prostate cancer: Biodistribution in humans and first evaluation of tumour lesions. Eur J Nucl Med Mol Imaging. 2013;40:486-495.

12. Herrmann K, Bluemel C, Weineisen M, et al. Biodistribution and radiation dosimetry for a probe targeting prostate-specific membrane antigen for imaging and therapy. J Nucl Med. 2015;56:855-861.

13. Afshar-Oromieh A, Hetzheim H, Kratochwil C, et al. The theranostic PSMA ligand PSMA-617 in the diagnosis of prostate cancer by PET/CT: Biodistribution in humans, radiation dosimetry, and first evaluation of tumor lesions. J Nucl Med. 2015;56:1697-1705.

14. Afshar-Oromieh A, Hetzheim H, Kubler W, et al. Radiation dosimetry of (68)ga-PSMA-11 (HBED-CC) and preliminary evaluation of optimal imaging timing. Eur J Nucl Med Mol Imaging. 2016;43:1611-1620.

15. Giesel FL, Will L, Lawal I, et al. Intraindividual comparison of (18)F-PSMA-1007 and (18)F-DCFPyL $\mathrm{PET} / \mathrm{CT}$ in the prospective evaluation of patients with newly diagnosed prostate carcinoma: A pilot study. J Nucl Med. 2018;59:1076-1080. 
16. Giesel FL, Hadaschik B, Cardinale J, et al. F-18 labelled PSMA-1007: Biodistribution, radiation dosimetry and histopathological validation of tumor lesions in prostate cancer patients. Eur J Nucl Med Mol Imaging. 2017;44:678-688.

17. Rowe SP, Pienta KJ, Pomper MG, Gorin MA. PSMA-RADS version 1.0: A step towards standardizing the interpretation and reporting of PSMA-targeted PET imaging studies. Eur Urol. 2018;73:485-487.

18. Landis JR, Koch GG. The measurement of observer agreement for categorical data. Biometrics. 1977;33:159-174.

19. Wondergem M, Jansen BHE, van der Zant FM, et al. Early lesion detection with (18)F-DCFPyL PET/CT in 248 patients with biochemically recurrent prostate cancer. Eur J Nucl Med Mol Imaging. 2019;46:1911-1918.

20. Dietlein F, Kobe C, Hohberg M, et al. Intraindividual comparison of (18)F-PSMA-1007 with renally excreted PSMA ligands for PSMA PET imaging in patients with relapsed prostate cancer. J Nucl Med. 2020;61:729-734.

21. Rauscher I, Krönke M, König M, et al. Matched-pair comparison of (68)ga-PSMA-11 PET/CT and (18)F-PSMA-1007 PET/CT: Frequency of pitfalls and detection efficacy in biochemical recurrence after radical prostatectomy. J Nucl Med. 2020;61:51-57.

22. Wondergem M, van der Zant FM, Knol RJJ, et al. (99m)tc-HDP bone scintigraphy and (18)Fsodiumfluoride PET/CT in primary staging of patients with prostate cancer. World J Urol. 2018;36:27-34. 
23. Sheikhbahaei S, Afshar-Oromieh A, Eiber M, et al. Pearls and pitfalls in clinical interpretation of prostate-specific membrane antigen (PSMA)-targeted PET imaging. Eur J Nucl Med Mol Imaging. 2017;44:2117-2136.

24. Cardinale J, Schäfer M, Benešová M, et al. Preclinical evaluation of (18)F-PSMA-1007, a new prostate-specific membrane antigen ligand for prostate cancer imaging. J Nucl Med. 2017;58:425-431. 

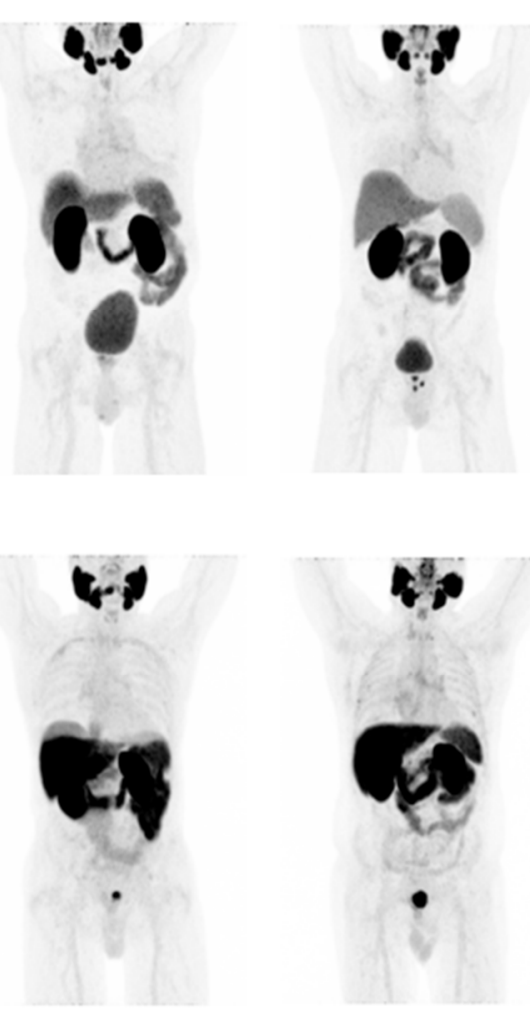

\section{${ }^{18}$ F-DCFPyL}
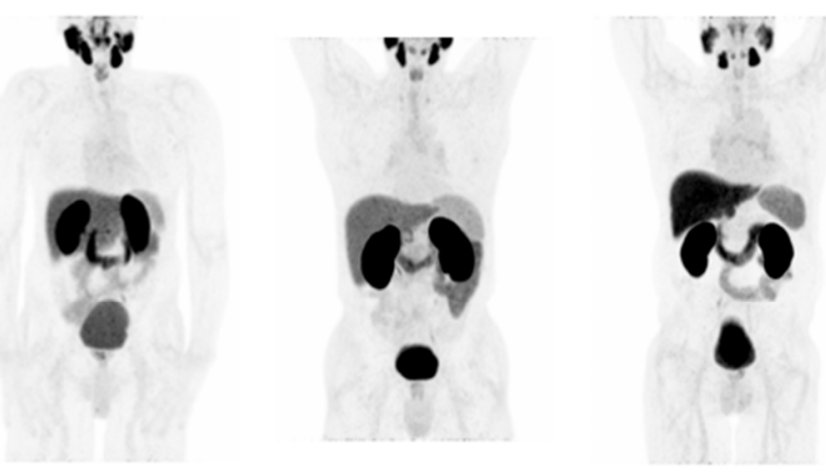

18F-PSMA-1007

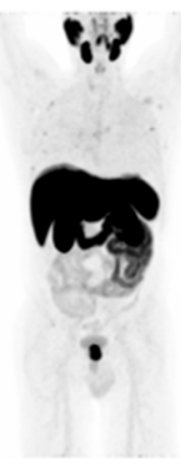

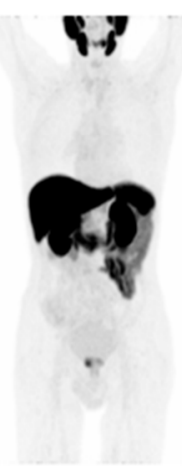

Figure 1. Distribution of DCFPyL and PSMA-1007 in patients imaged for primary screening of high-risk prostate cancer, all scored as negative for bone and lymph node metastases by two experienced readers. Maximum Intensity Projection images scaled to SUV-body weight 0.0-10.0. Lower physiological DCFPyL uptake is observed in liver, spleen and bone marrow. Lower DCFPyL uptake is observed in the primary prostate tumor. Low PSMA-1007 activity is seen in the urinary tract and trachea. PSMA-1007 uptake skeletal uptake is heterogenous between patients; none tot diffuse uptake in bone marrow is observed and variable patterns of irregular PSMA-1007 uptake is observed in the ribs of several patients. Higher PSMA-1007 activity in the bile ducts and gall bladder is not appreciated due to scaling. 


\begin{tabular}{|l|l|l|l|l|}
\hline \multicolumn{4}{|l|}{ Total cohort } \\
\hline & WCFPyL & \multicolumn{2}{l|}{ PSMA-1007 } \\
\hline & Weighted k & $\begin{array}{l}\text { Agreement } \\
\text { (\%) }\end{array}$ & Weighted k & $\begin{array}{l}\text { Agreement } \\
(\%)\end{array}$ \\
\hline Prostate & 0.925 & 95 & 0.738 & 90 \\
\hline Lymph nodes & & & & \\
\hline Inguinal & 0.265 & 96 & 0.746 & 99 \\
\hline Pelvis & 0.915 & 95 & 0.841 & 89 \\
\hline Abdomen & 0.848 & 93 & 0.833 & 93 \\
\hline Mediastinum & 0.852 & 96 & 0.673 & 94 \\
\hline Axillae & 1.000 & 100 & 1.000 & 100 \\
\hline Neck & 0.773 & 95 & 0.952 & 98 \\
\hline Whole body & 0.916 & 94 & 0.825 & 88 \\
\hline Bone & & & & \\
\hline Pelvis & 0.895 & 95 & 0.823 & 86 \\
\hline Spine & 0.893 & 95 & 0.912 & 93 \\
\hline Thorax & 0.925 & 95 & 0.706 & 74 \\
\hline Extremities & 1.000 & 100 & 0.847 & 94 \\
\hline Whole skeleton & 0.868 & 93 & 0.659 & 70 \\
\hline
\end{tabular}

Figure 2. Inter-reader agreement reflected by weighted Cohen's kappa (weighted $\mathrm{k}$ ) and by percentage for both DCFPyL and PSMA-1007. Degree of agreement; dark green: very good, light green: good, orange: moderate 


\section{Thorax}

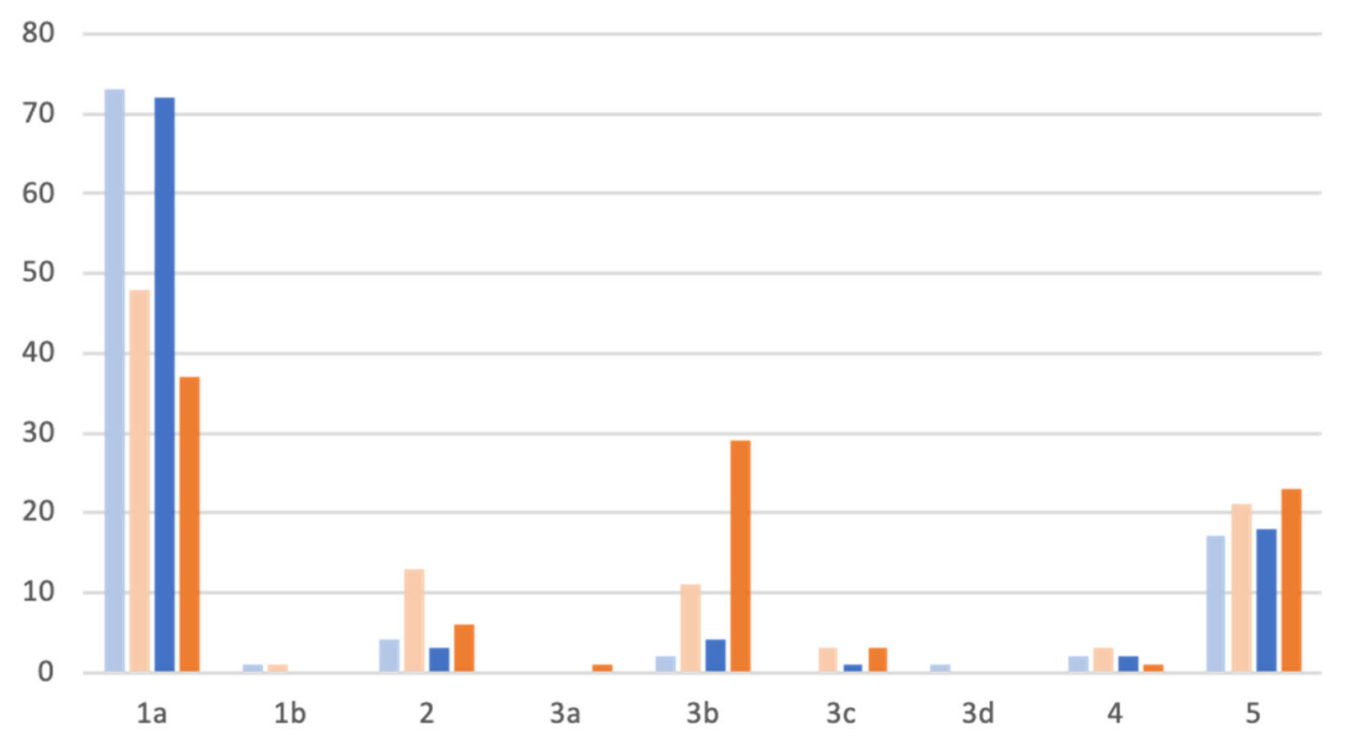

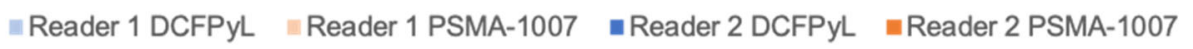

\section{Whole body}

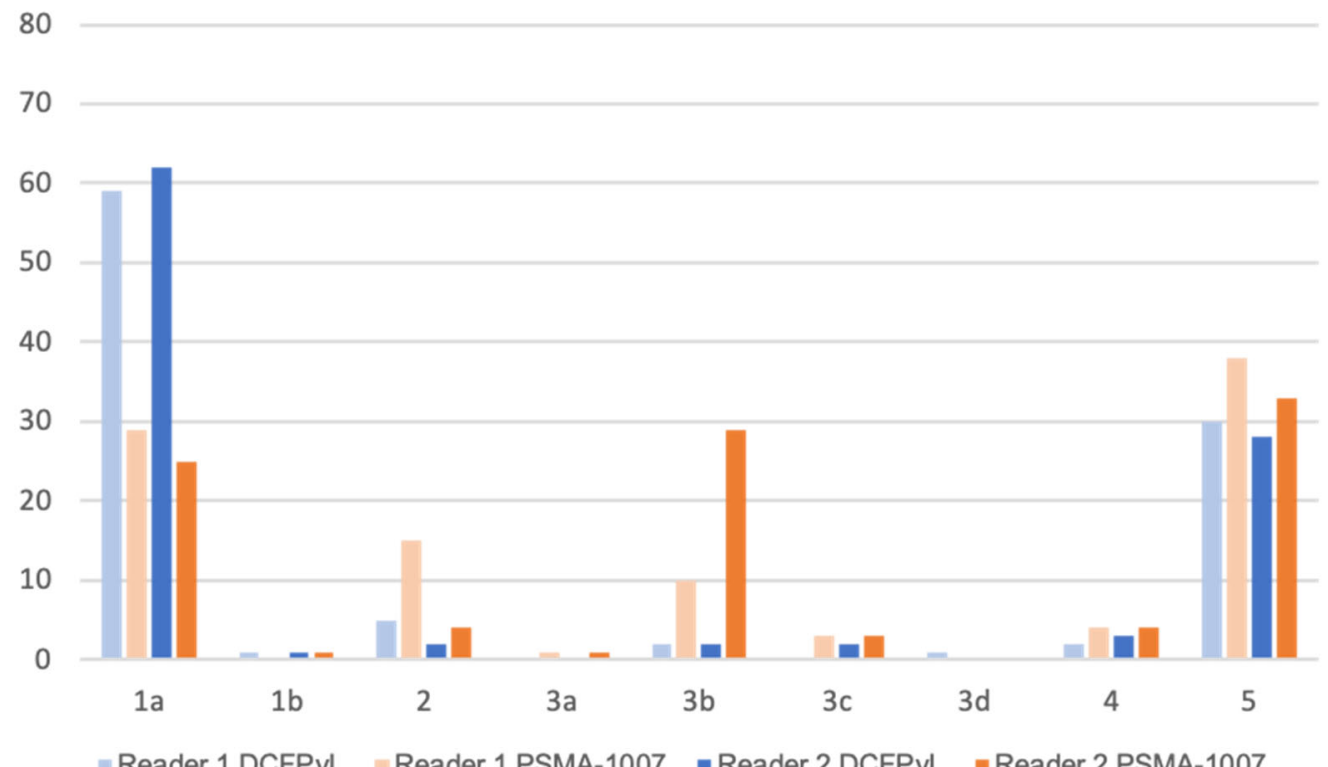

Figure 3. Bar graph for bone lesions in the thorax and whole body. For PSMA-1007 both readers score statistically significantly more equivocal lesions ( $3 a, 3 b, 3 c$ or $3 d$ ) and less benign lesions ( $1 a, 1 b$ and 2$)$. 

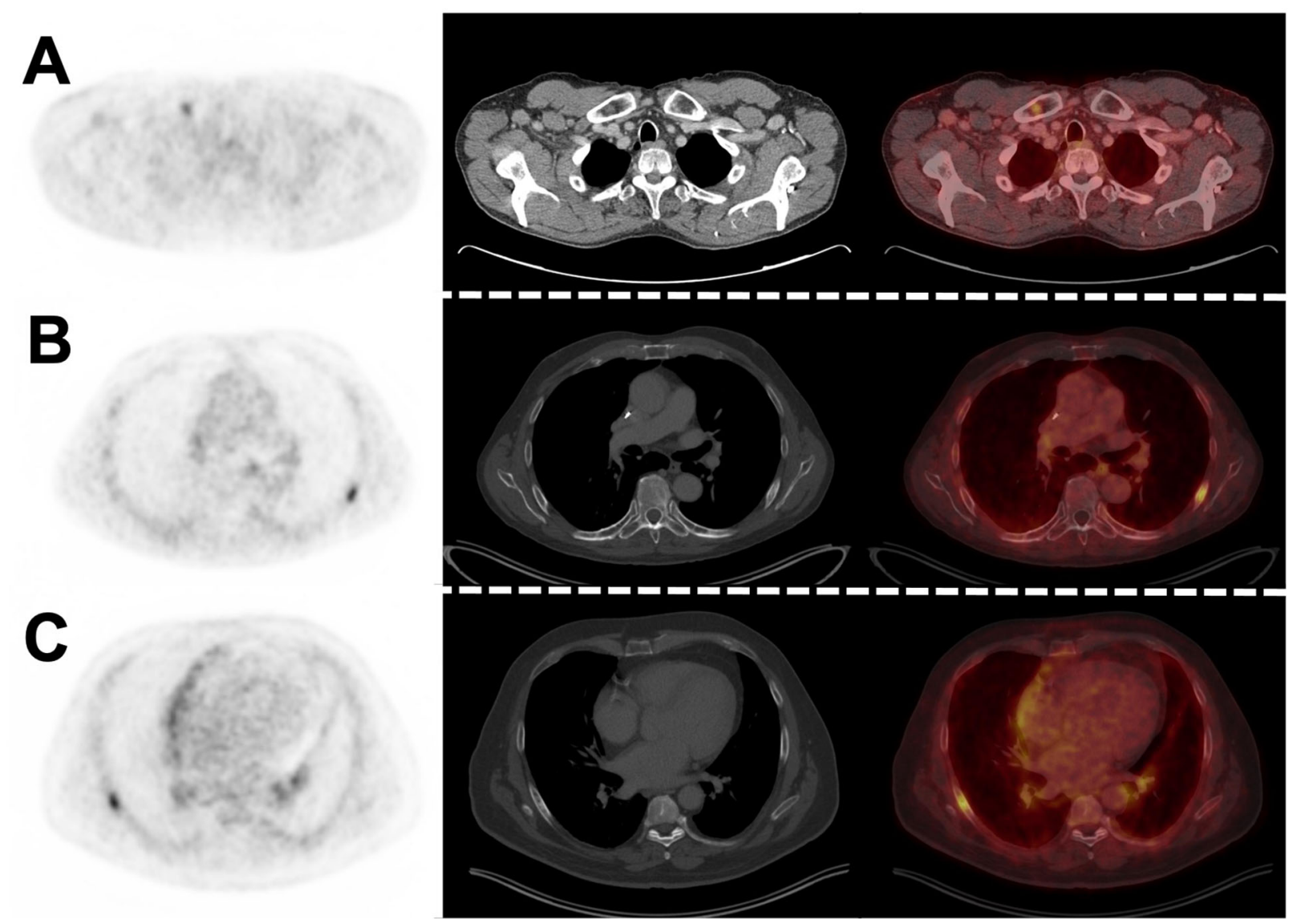

Figure 4. Equivocal bone lesions in right clavicula (patient $A$ ) and ribs (patient $B$ and $C$ )of clinical relevance on PSMA-1007 PET/CT for primary staging in three patients. A: cT3a Gleason 7, iPSA $15.2 \mathrm{ng} / \mathrm{ml}$; B: cT2 Gleason 8 iPSA $8.0 \mathrm{ng} / \mathrm{ml}$; C: cT3a Gleason 6 iPSA $3.7 \mathrm{ng} / \mathrm{ml}$. Additional MRI could not exclude bone metastasis in patient A. No further imaging was performed in patient $B$ and $C$. Histopathological biopsy revealed no malignancy in all three cases. A: aspecific necrosis, B: aspecific lytic bone lesion with reactive changes, C: normal bone tissue. Ultimately, all three patients were staged NOMO ( $8^{\text {th }}$ TNM-classification, American Joint Committee on Cancer). 
Table 1. Patients' characteristics.

\begin{tabular}{|c|c|c|}
\hline & DCFPyL & PSMA-1007 \\
\hline \multicolumn{3}{|l|}{ All patients } \\
\hline $\mathrm{N}$ & 120 & 120 \\
\hline PSA ( $\mu \mathrm{g} / \mathrm{ml}$ ) (median; range) & $11.6 ; 0.1-558$ & $12.0 ; 0.1-577$ \\
\hline \multicolumn{3}{|l|}{ Primary staging } \\
\hline $\mathrm{N}$ & 69 & 69 \\
\hline PSA ( $\mu \mathrm{g} / \mathrm{ml})$ (median; range) & $14.8 ; 3.2-558$ & $14.7 ; 2.4-577$ \\
\hline \multicolumn{3}{|l|}{ Gleason score $^{1}$} \\
\hline$\leq 7 a$ & 17 & 8 \\
\hline$\geq 7 b$ & 51 & 58 \\
\hline Unknown & 1 & 4 \\
\hline \multicolumn{3}{|l|}{ T-stage $^{2}$} \\
\hline $\mathrm{T} 1$ & 23 & 15 \\
\hline $\mathrm{T} 2$ & 25 & 27 \\
\hline T3 & 16 & 22 \\
\hline T4 & 0 & 4 \\
\hline Unknown & 5 & 1 \\
\hline ADT & 0 & 0 \\
\hline \multicolumn{3}{|l|}{ Biochemical recurrence } \\
\hline $\mathrm{N}$ & 21 & 21 \\
\hline PSA ( $\mu \mathrm{g} / \mathrm{ml})$ (median; range) & $2.4 ; 0.4-7.9$ & $2.4 ; 0.4-7.8$ \\
\hline \multicolumn{3}{|l|}{ Previous therapy } \\
\hline Prostatectomy & 7 & 7 \\
\hline Radiation therapy & 12 & 12 \\
\hline Brachytherapy & 2 & 2 \\
\hline $\mathrm{ADT}^{3}$ & 1 & 2 \\
\hline \multicolumn{3}{|l|}{ Follow-up systemic treatment } \\
\hline $\mathrm{N}$ & 30 & 30 \\
\hline PSA ( $\mu \mathrm{g} / \mathrm{ml}$ ) (median; range) & $24.8 ; 0.1-385.0$ & $26.3 ; 0.1-369.0$ \\
\hline ADT & 30 & 30 \\
\hline
\end{tabular}

1. $p=0.402$ and $^{2 .} p=0.009$ (Mann-Whitney $U$ test). ${ }^{3 .}$ ADT only temporarily after radiation therapy with curative intention. 
Table 2. Detection rates $(n /(\%))$ of suspected lesions and equivocal lesions and testing of equality for detection of suspected lesions between DCFPyL and PSMA-1007 (Mann-Whitney U test) for the total cohort.

\begin{tabular}{|c|c|c|c|c|c|c|c|c|c|c|c|}
\hline & & Reader 1 & & & & & Reader 2 & & & & \\
\hline & & DCFPyL & & $\begin{array}{l}\text { PSMA- } \\
1007\end{array}$ & & $p$ & DCFPyL & & $\begin{array}{l}\text { PSMA- } \\
1007\end{array}$ & & $p$ \\
\hline & & $\begin{array}{l}\text { Suspected } \\
\text { malignant }\end{array}$ & Equivocal & $\begin{array}{l}\text { Suspected } \\
\text { malignant }\end{array}$ & Equivocal & & $\begin{array}{l}\text { Suspected } \\
\text { malignant }\end{array}$ & Equivocal & $\begin{array}{l}\text { Suspected } \\
\text { malignant }\end{array}$ & Equivocal & \\
\hline Prostate & & $86 /(72)$ & $4 /(3)$ & $98 /(82)$ & $7 /(6)$ & $0.045^{*}$ & $86 /(72)$ & $5 /(4)$ & $102 /(85)$ & $1 /(1)$ & $0.015^{*}$ \\
\hline \multirow[t]{7}{*}{ Lymph nodes } & Inguinal & $4 /(3)$ & $0 /(0)$ & $2 /(2)$ & $0 /(0)$ & 0.409 & $4 /(3)$ & $0 /(0)$ & $1 /(1)$ & $2 /(2)$ & 0.984 \\
\hline & Pelvis & $52 /(43)$ & $2 /(2)$ & $55 /(46)$ & $4 /(3)$ & 0.409 & $52 /(43)$ & $2 /(2)$ & $55 /(46)$ & $5 /(4)$ & 0.460 \\
\hline & Abdomen & $36 /(30)$ & $0 /(0)$ & $31 /(26)$ & $0 /(0)$ & 0.473 & $41 /(34)$ & $2 /(2)$ & $34 /(28)$ & $2 /(2)$ & 0.331 \\
\hline & Mediastinum & $12 /(10)$ & $0 /(0)$ & $7 /(6)$ & $4 /(3)$ & 0.760 & $13 /(11)$ & $4 /(3)$ & $10 /(8)$ & $1 /(1)$ & 0.332 \\
\hline & Axillae & $6 /(5)$ & $0 /(0)$ & $1 /(1)$ & $0 /(0)$ & 0.056 & $6 /(5)$ & $0 /(0)$ & $1 /(1)$ & $0 /(0)$ & 0.056 \\
\hline & Neck & $12 /(10)$ & $0 /(0)$ & $11 /(9)$ & $1 /(1)$ & 0.983 & $18 /(15)$ & $0 /(0)$ & $11 /(9)$ & $1 /(1)$ & 0.231 \\
\hline & Whole body & $52 /(43)$ & $2 /(2)$ & $56 /(47)$ & $5 /(4)$ & 0.463 & $55 /(46)$ & $4 /(3)$ & $58 /(48)$ & $6 /(5)$ & 0.691 \\
\hline \multirow[t]{5}{*}{ Bone } & Pelvis & $32 /(27)$ & $2 /(2)$ & $44 /(37)$ & $5 /(4)$ & 0.053 & $32 /(27)$ & $0 /(0)$ & $40 /(33)$ & $13 /(11)$ & $0.027^{*}$ \\
\hline & Spine & $28 /(23)$ & $2 /(2)$ & $34 /(28)$ & $4 /(3)$ & 0.215 & $26 /(22)$ & $1 /(1)$ & $34 /(28)$ & $10 /(8)$ & 0.060 \\
\hline & Thorax & $23 /(19)$ & $4 /(3)$ & $29 /(24)$ & $17 /(14)$ & $0.015^{*}$ & $24 /(20)$ & $5 /(4)$ & $28 /(23)$ & $40 /(33)$ & $<0.001^{*}$ \\
\hline & Extremities & $22 /(18)$ & $0 /(0)$ & $18 /(15)$ & $4 /(3)$ & 0.929 & $22 /(18)$ & $0 /(0)$ & $18 /(15)$ & $4 /(3)$ & 0.929 \\
\hline & Whole skeleton & $40 /(33)$ & $4 /(3)$ & $50 /(42)$ & $17 /(14)$ & $0.009 *$ & $36 /(30)$ & $5 /(4)$ & $44 /(37)$ & $40 /(33)$ & $<0.001^{*}$ \\
\hline \multirow[t]{4}{*}{ Other } & Lung & $1 /(1)$ & $4 /(3)$ & $1 /(1)$ & $7 /(6)$ & 0.389 & $1 /(1)$ & $6 /(5)$ & $2 /(2)$ & $6 /(5)$ & 0.800 \\
\hline & Liver & $1 /(1)$ & $0 /(0)$ & $2 /(2)$ & $1 /(1)$ & 0.312 & $0 /(0)$ & $1 /(1)$ & $2 /(2)$ & $1 /(1)$ & 0.318 \\
\hline & Funiculus & $0 /(0)$ & $2 /(2)$ & $0 /(0)$ & $0 /(1)$ & 0.156 & $2 /(2)$ & $0 /(0)$ & $0 /(0)$ & $0 /(0)$ & 0.800 \\
\hline & Adrenals & $1 /(1)$ & $0 /(0)$ & $1 /(1)$ & $1 /(1)$ & 0.559 & $0 /(0)$ & $0 /(0)$ & $0 /(0)$ & $0 /(0)$ & 1.000 \\
\hline
\end{tabular}




\begin{tabular}{|l|l|r|r|r|r|r|r|r|r|r|r|}
\hline & Pleural & $0 /(0)$ & $0 /(0)$ & $0 /(0)$ & $1 /(1)$ & 0.317 & $0 /(0)$ & $0 /(0)$ & $0 /(0)$ & $1 /(1)$ & 0.317 \\
\hline & Thyroid & $0 /(0)$ & $0 /(0)$ & $0 /(0)$ & $0 /(0)$ & 1.000 & $0 /(0)$ & $0 /(0)$ & $0 /(0)$ & $1 /(1)$ & 0.317 \\
\hline & All other & $3 /(3)$ & $6 /(5)$ & $4 /(3)$ & $10 /(8)$ & 0.270 & $3 /(3)$ & $7 /(6)$ & $4 /(3)$ & $9 /(8)$ & 0.513 \\
\hline
\end{tabular}

Suspected malignant PSMA-RADS 4 and 5, equivocal PSMA-RADS 3a, 3b, 3c, and 3d. *Statistically significant findings $(p<0.05)$. 
Table 3. Detection rates $(n /(\%))$ of suspected lesions and equivocal lesions and testing of equality for detection of suspected lesions between DCFPyL and PSMA-1007 (Mann-Whitney U test) according to disease stage categories primary staging, biochemical recurrence and follow-up of systemic therapies.

\begin{tabular}{|c|c|c|c|c|c|c|c|c|c|c|c|c|c|c|c|c|}
\hline & & $\begin{array}{l}\text { Prim } \\
(n=69)\end{array}$ & & & & & $\begin{array}{c}\text { BCR } \\
(n=21)\end{array}$ & & & & & $\begin{array}{l}\text { FU } \\
(n=30)\end{array}$ & & & & \\
\hline & & DCFPyL & & $\begin{array}{l}\text { PSMA- } \\
1007\end{array}$ & & & DCFPyL & & $\begin{array}{l}\text { PSMA- } \\
1007\end{array}$ & & & DCFPyL & & $\begin{array}{l}\text { PSMA- } \\
1007\end{array}$ & & \\
\hline & & $\begin{array}{l}\text { Suspected } \\
\text { malignant }\end{array}$ & Equivocal & $\begin{array}{l}\text { Suspected } \\
\text { malignant } \\
\end{array}$ & Equivocal & $p$ & $\begin{array}{l}\text { Suspected } \\
\text { malignant }\end{array}$ & Equivocal & $\begin{array}{l}\text { Suspected } \\
\text { malignant } \\
\end{array}$ & Equivocal & $p$ & $\begin{array}{l}\text { Suspected } \\
\text { malignant } \\
\end{array}$ & Equivocal & $\begin{array}{l}\text { Suspected } \\
\text { malignant } \\
\end{array}$ & Equivocal & $p$ \\
\hline \multirow[t]{3}{*}{$\begin{array}{l}\text { Reader } \\
1 \\
\end{array}$} & Prostate & $66 /(96)$ & $2 /(3)$ & $67 /(97)$ & $2 /(3)$ & 0.669 & $6 /(29)$ & $0 /(0)$ & $13 /(62)$ & $3 /(14)$ & $0.007^{*}$ & $14 /(47)$ & $2 /(7)$ & $9 /(30)$ & (13) & 0.186 \\
\hline & $\begin{array}{r}\text { Lymph } \\
\text { nodes }\end{array}$ & $24 /(35)$ & $1 /(1)$ & $27 /(39)$ & $4 /(6)$ & 0.393 & $11 /(52)$ & $1 /(5)$ & $9 /(43)$ & $1 /(5)$ & 0.532 & $17 /(57)$ & $0 /(0)$ & $20 /(67)$ & (0) & 0.430 \\
\hline & $\begin{array}{r}\text { Whole } \\
\text { skeleton }\end{array}$ & $17 /(25)$ & $1 /(1)$ & $20 /(29)$ & $11 /(16)$ & 0.065 & $2 /(10)$ & $1 /(5)$ & $6 /(29)$ & $5 /(24)$ & $0.013 *$ & $20 /(67)$ & $1 /(3)$ & $24 /(80)$ & (3) & 0.234 \\
\hline \multirow[t]{3}{*}{$\begin{array}{l}\text { Reader } \\
2 \\
\end{array}$} & Prostate & $67 /(97)$ & $2 /(3)$ & $68 /(99)$ & $1 /(1)$ & 0.572 & $6 /(29)$ & $1 /(5)$ & $13 /(62)$ & $1 /(5)$ & 0.029* & $13 /(43)$ & $2 /(7)$ & $21 /(70)$ & (0) & 0.059 \\
\hline & $\begin{array}{r}\text { Lymph } \\
\text { nodes } \\
\end{array}$ & $25 /(36)$ & $4 /(6)$ & $28 /(41)$ & $4 /(6)$ & 0.595 & $11 /(52)$ & $0 /(0)$ & $7 /(33)$ & $1 /(5)$ & 0.285 & $19 /(63)$ & $0 /(0)$ & $22 /(73)$ & (3) & 0.356 \\
\hline & $\begin{array}{r}\text { Whole } \\
\text { skeleton }\end{array}$ & $15 /(22)$ & $2 /(3)$ & $15 /(22)$ & $27 /(39)$ & $0.001 *$ & $3 /(14)$ & $2 /(10)$ & $7 /(33)$ & $9 /(43)$ & $0.003 *$ & $18 /(60)$ & $1 /(3)$ & $22 /(73)$ & (13) & 0.154 \\
\hline
\end{tabular}

Prim: primary staging; BCR: biochemical recurrence; FU: follow up of systemic therapies. Suspected malignant PSMA-RADS 4 and 5, equivocal

PSMA-RADS 3a, 3b, 3c, and 3d. *Statistically significant findings $(p<0.05)$. 


\section{Prostate / Prostatic region}

60

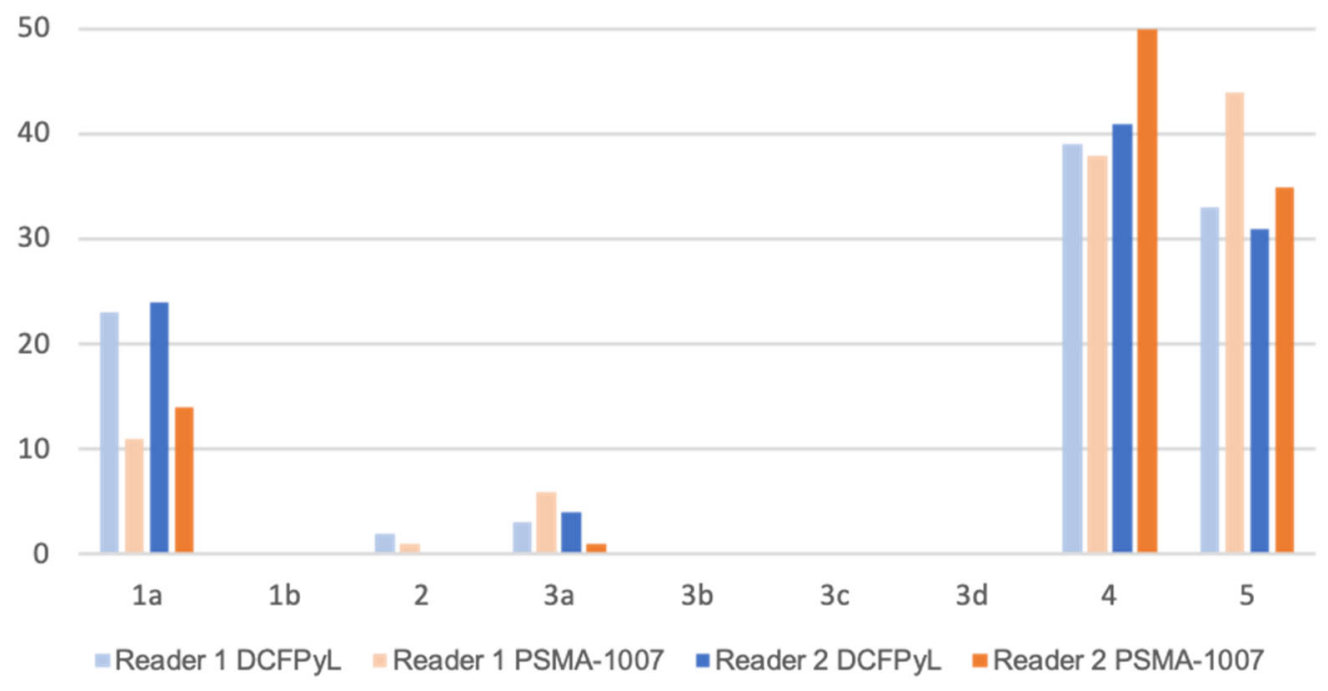

Supplement 1. Bar graph for prostate / prostatic region for whole cohort and sub cohort of biochemical recurrence. Both readers found more suspected prostate lesions with PSMA-1007 (reader 1 p=0.045; reader 2 $\mathrm{p}=0.015)$. 


\section{Lymph nodes}

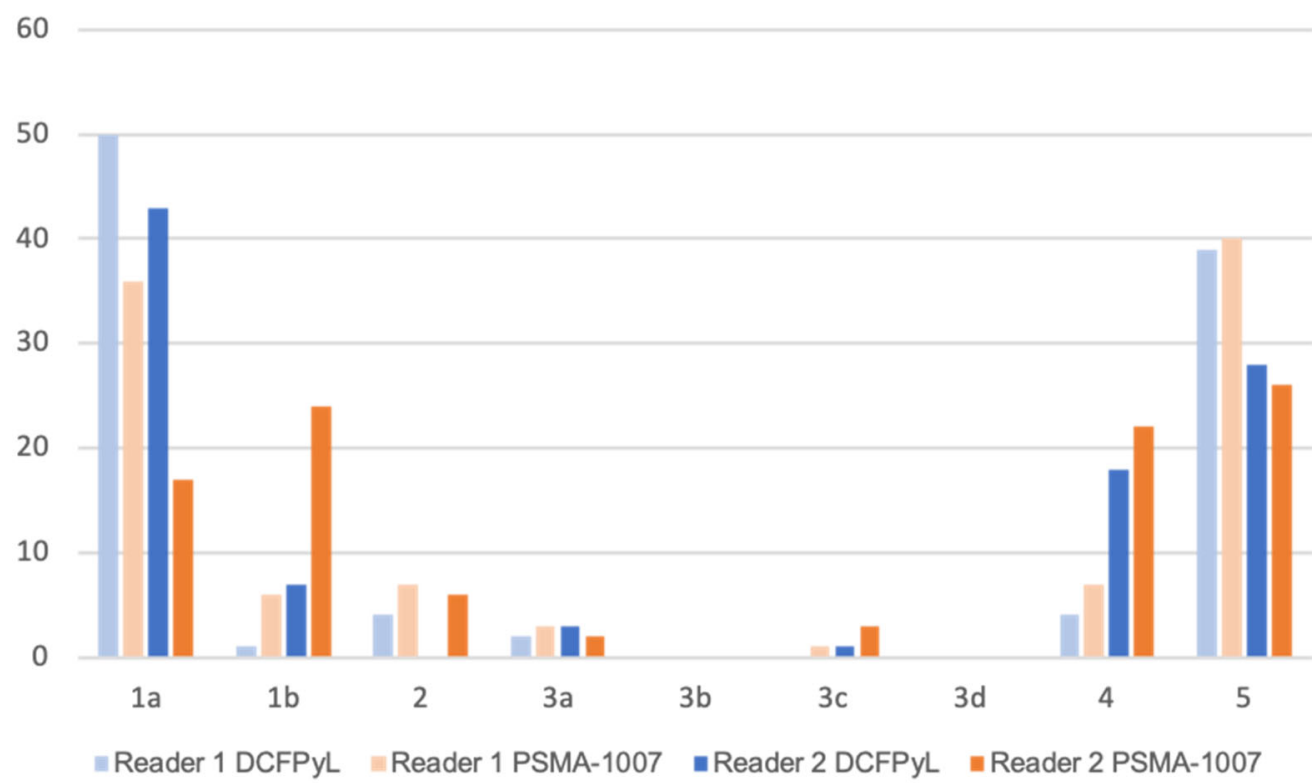

Supplement 2. Bar graph for lymph nodes in the whole body. For PSMA-1007 both readers score relatively more nodes with low level uptake (classified $1 \mathrm{~b}$ or 2 ). 
${ }^{18}$ F-DCFPyL
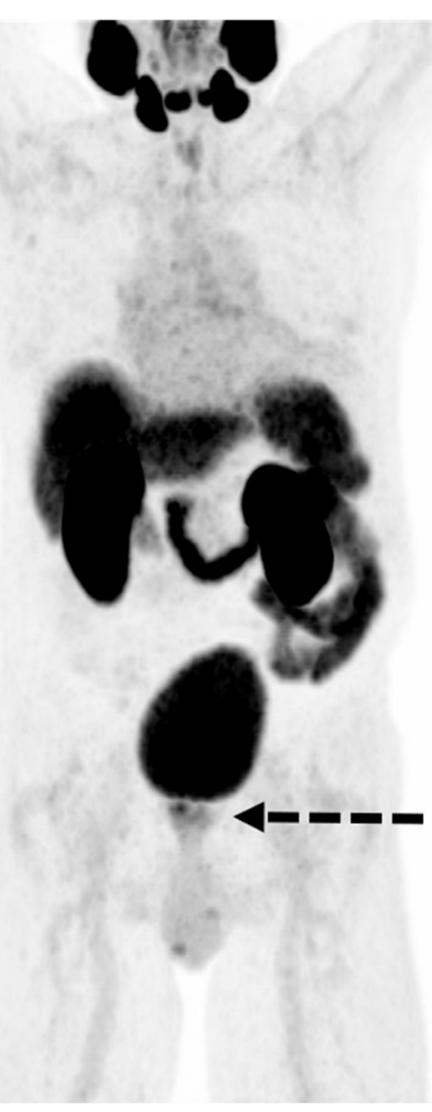

${ }^{18} \mathrm{~F}-\mathrm{PSMA}-1007$

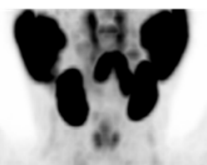

Equivocal bone uptake

may interfere with

detection of bone

$-m-$

metastases

Urinary excration may interfere with detection of prostate lesions

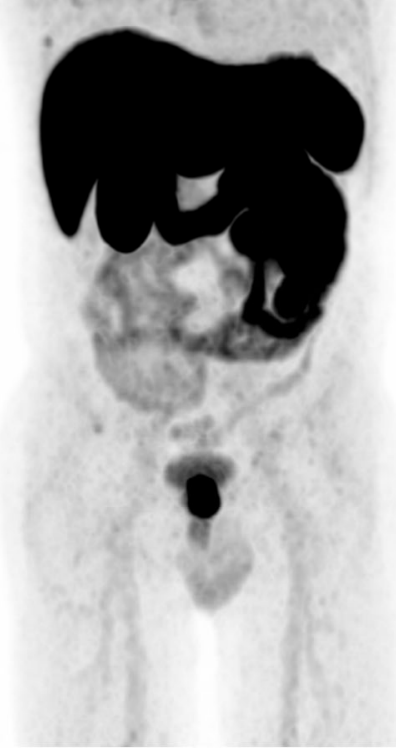

Equivocal bone lesion;

biopsy confirmed benign etiology

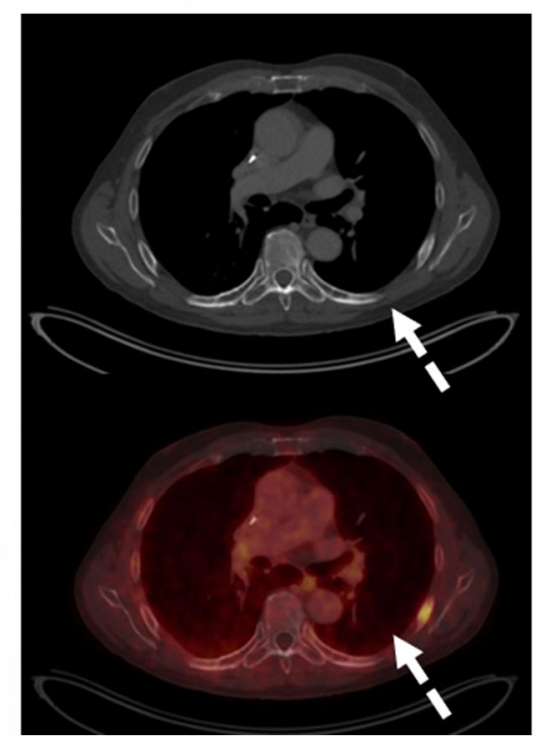

